\title{
Theory of fast optical spin rotation in a quantum dot based on geometric phases and trapped states
}

\author{
Sophia E. Economou and T. L. Reinecke \\ Naval Research Laboratory, Washington, DC 20375, USA
}

(Dated: October 28, 2018)

\begin{abstract}
A method is proposed for the optical rotation of the spin of an electron in a quantum dot using excited trion states to implement operations up to two orders of magnitude faster than those of most existing proposals. Key ingredients are the geometric phase induced by $2 \pi$ hyperbolic secant pulses, use of coherently trapped states and use of naturally dark states. Our proposal covers a wide variety of quantum dots by addressing different parameter regimes. In one case the treatment provides an exact solution to the three-level system. Numerical simulations with typical parameters for InAs self-assembled quantum dots, including their dissipative dynamics, give fidelities of the operations in excess of $99 \%$.
\end{abstract}

All-optically controlled spins in quantum dots (QDs) provide an attractive proposal for quantum information processing (QIP) [1]. This proposal combines the merits of solid state, such as integrability with existing semiconductor technology, with the speed and high degree of control of lasers. Among the requirements for QIP [2], optical preparation [3] and measurement [4, 5] of the spin qubit have been achieved, and the spin coherence time has been measured to be at least $3 \mu \mathrm{s}[\underline{6}]$. At the heart of this QIP approach are optical spin rotations that constitute the quantum gates by which information is manipulated. This key step toward the development of optical QIP with spins in QDs has not yet been demonstrated experimentally, although several proposals have been given 17, 8, 9, 10, 11]. Most of the latter employ the adiabatic approximation either through adiabatic elimination of an excited state [7, 8] or through Stimulated Raman Adiabatic Passage [9, 10], and thus they rely on adiabatic pulses that limit the speed of the gates. A method for fast optical spin rotations about a single axis (the optical axis) was proposed [1]], but rotations about arbitrary axes are required for QIP.

Here we present a proposal for achieving arbitrary coherent spin rotations without making use of an adiabatic approximation. We show that for realistic QD systems our method attains high (up to $99.99 \%$ ) fidelity gates operating up to two orders of magnitude faster than other proposals. Our approach involves naturally dark or coherently trapped states (depending on the system parameters involved) combined with novel use of the geometric phase analysis [11 for the hyperbolic secant pulses of Rosen and Zener [12].

These sech pulses have the remarkable property of rendering the resulting time dependent Schrödinger equation for a two-level system analytically solvable. A pulse area can be defined for them independent of the detuning, an asset that led to the celebrated phenomenon of self-induced transparency [13] for $2 \pi$ pulses. Such pulses propagate virtually unattenuated through resonant optical media, returning the individual two-level systems to their initial states. This is a highly attractive feature for the current QIP proposal because the information must be returned and stored in the spin subspace after it is manipulated. A less explored effect is that after the passage of the $2 \pi$ sech pulse the state also acquires a geometric phase, for which we obtained an analytic expression [11]. This phase is global (an overall phase to the quantum state) for a two-level system. It becomes crucial when a third state that does not couple to the laser is present, and it is one of the key ingredients of the present proposal.

The energy levels and selection rules of the spin system in our approach are shown in Fig. 1. An external magnetic field $\mathrm{B}$ along the in-plane $(x)$ direction defines the quantization axis for the electron spin; the optical axis, which coincides with the growth axis of the QD, defines the $z$ direction. The intermediate states are the so-called trion states, which are bound states of an electron and an exciton, the latter created in the QD by the laser. The angular momentum of the single electron levels comes from its spin. The hole has a total angular momentum of $3 / 2$, and the relevant excited states are the $m_{j}= \pm 3 / 2$ ('heavy hole') trion states quantized along $z$. In typical QDs, the $m_{j}= \pm 1 / 2$ ('light hole') states are separated by more than $20 \mathrm{meV}$ from the $m_{j}= \pm 3 / 2$ states due to confinement, and they are not included here. For a magnetic field along $x$ the trion energy eigenstates, $\left|T_{x}\right\rangle,\left|T_{\bar{x}}\right\rangle$, are linear combinations of the heavy hole trions. Fig. 1 shows the optical selection rules: the transitions $|x\rangle \rightarrow\left|T_{x}\right\rangle$ and $|\bar{x}\rangle \rightarrow\left|T_{\bar{x}}\right\rangle\left(|x\rangle \rightarrow\left|T_{\bar{x}}\right\rangle\right.$ and $\left.|\bar{x}\rangle \rightarrow\left|T_{x}\right\rangle\right)$ are coupled by linearly polarized light $\pi_{x}$ $\left(\pi_{y}\right)$. The Zeeman splittings for the electron and the trion are $2 \omega_{e}$ and $2 \omega_{h}$ respectively. The trion splitting is determined by the hole $g$ factor because the electrons are in the same orbital state of the QD (a spin singlet) and do not contribute to the total trion spin.

Our proposal is based on the observation that from the four-level system of Fig. 1, different two-level systems can be selected by an appropriate choice of laser polarization without the need for frequency selectivity. In our approach all four states participate in the rotation scheme. For each of the decoupled two-level systems, a 


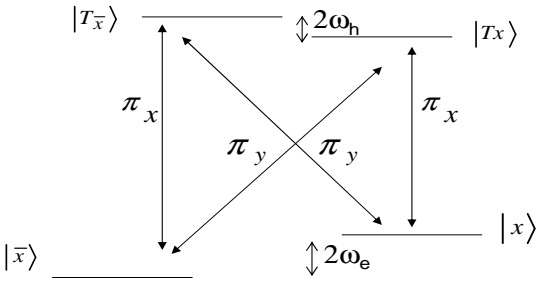

FIG. 1: Energy levels of the four-state system, which is comprised of the two electron spin eigenstates of $\sigma_{x},\{|x\rangle,|\bar{x}\rangle\}$ and the two trion spin states. Linearly polarized light $x(y)$ denoted by $\pi_{x}\left(\pi_{y}\right)$ induces only the indicated transitions.

$2 \pi$ sech pulse will be used to induce the desired phase in the ground state. It is well known that given arbitrary rotations by two axes any rotation can be implemented as a composite rotation. Here we design rotations about the $z$ and the $x$ axes by arbitrary angles and compose general rotations from them.

(a) Rotations about $z$ - We showed earlier [11] that broadband circularly polarized pulses (here determined by the bandwidth $\left.\beta_{z} \gg 2\left(\omega_{e}+\omega_{h}\right)\right)$ allow the four-level system in Fig. 1 to be treated as a two-level system (plus two uncoupled levels): During the ultrafast pulse the spin and trion precessions are 'frozen'. The phase gates of Ref. [11] are used for $z$ rotations. The angle of rotation is

$$
\phi_{z}=2 \arctan \left(\beta_{z} / \Delta\right)
$$

where $\Delta$ is the detuning. The fidelity and purity of the $z$ operations both increase as the pulses become faster [11]. For a subpicosecond pulse, we found the fidelity to be as high as $99.99 \%$.

(b) Rotations about $x$ - As illustrated in Fig. 1 by choosing linearly polarized light $\pi_{x}$, we reduce the four-level system to two independent two-level systems, $\left\{|x\rangle,\left|T_{x}\right\rangle\right\}$ and $\left\{|\bar{x}\rangle,\left|T_{\bar{x}}\right\rangle\right\}$. A $\pi_{x}$ linearly polarized $2 \pi$ sech pulse with bandwidth $\beta_{x}$ is used here, which we allow to act on both transitions and which is detuned by $\Delta_{1}\left(\Delta_{2}\right)$ for transition $|x\rangle \leftrightarrow\left|T_{x}\right\rangle\left(|\bar{x}\rangle \leftrightarrow\left|T_{\bar{x}}\right\rangle\right)$. Each two-level system acquires a phase (Eq. 1) determined by its detuning. The relative phase determines the angle of rotation about the $x$ axis, $\phi_{x}=\phi_{1}-\phi_{2}$, which is

$$
\phi_{x}=2 \arctan \frac{\beta_{x}\left(\Delta_{1}-\Delta_{2}\right)}{\Delta_{1} \Delta_{2}+\beta_{x}^{2}}
$$

in terms of pulse and system parameters. From Eq. (2) it is clear that a requirement for a $\pi$ rotation is $\Delta_{1} \Delta_{2}<0$. This can be understood intuitively: If the laser is detuned positively (or negatively) for both transitions, then the phases will have the same sign, so there is an upper limit to the obtainable angle of rotation. Centering the

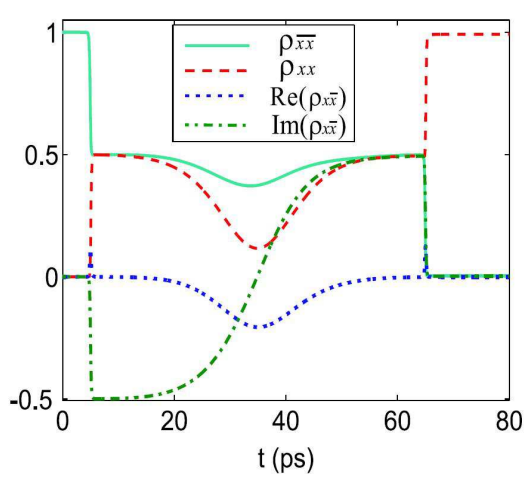

FIG. 2: (Color online) The matrix elements of the spin density matrix, $\rho$, for the rotation $R_{y}(\pi)$. The red (dashed) line is $\rho_{x x}$, the cyan (solid) line is $\rho_{\bar{x} \bar{x}}$, the blue (dotted) line is $\operatorname{Re}\left(\rho_{x \bar{x}}\right)$, and the green (dashed-dotted) line is $\operatorname{Im}\left(\rho_{x \bar{x}}\right)$, and $\rho$ is the density matrix in the interaction picture (free spin precession is removed). The duration of this composite gate is about 60 ps.

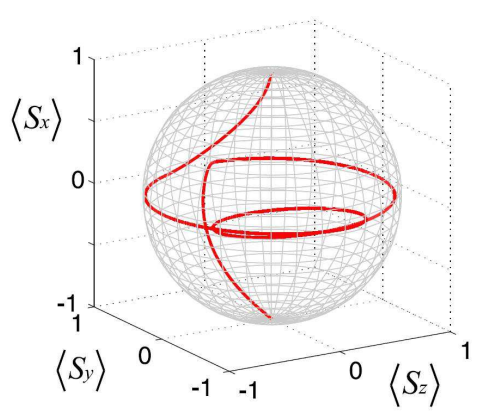

FIG. 3: (Color online) Bloch sphere representation of the composite spin rotation $R_{y}(\pi)$ acting on the initial spin state $|\bar{x}\rangle$, i.e., $\left\langle S_{x}\right\rangle=-1$.

laser frequency between the two transitions gives rotation angles up to $\pi$ provided that $\beta_{x}$ is chosen properly. Therefore for a $\pi$ rotation there is an upper bound to the bandwidth $\beta_{x}$, which makes rotations about $x$ slower than those about $z$.

(c) Rotations about other axes- Finally, by combining the above rotations about $x$ and $z$ we can implement any rotation. For example, rotations about $y$ can be realized by $R_{y}(\phi)=R_{z}^{\dagger}(\pi / 2) R_{x}(\phi) R_{z}(\pi / 2)$ or alternatively by sandwiching a $z$ rotation between two $x$ rotations.

The imperfections in these gates come predominately from trion decay and spin precession during $z$ rotations. These effects are taken into account in our calculations of the fidelity by numerical solution of the Liouville equation for the density matrix. The fidelity is a measure of how well the gate is implemented and is defined as $\mathcal{F}(U)=\overline{\left|\left\langle\Psi\left|U^{\dagger} U_{i d}\right| \Psi\right\rangle\right|^{2}}$, where $U_{i d}$ is the target operation, $U$ is the actual operation, and the average is taken over all input spin states [14]. The purity of an operation 
is $\mathcal{P}=\overline{\operatorname{Tr} \rho^{2}}$, where $\rho$ is the spin density matrix after the rotation.

We have made calculations of the fidelity and purity of gates using parameters appropriate for realistic selfassembled InAs QDs: $\omega_{e}=0.05 \mathrm{meV}, \omega_{h}=0.033 \mathrm{meV}$ (corresponding to $\mathrm{B} \sim 8 \mathrm{~T}$ ), $\beta_{x}=0.073 \mathrm{meV}$ for $\pi$ rotations, $\beta_{x}=0.113 \mathrm{meV}$ for $\pi / 2$-rotations, $\beta_{z}=4$ meV, and trion lifetime $\tau_{t}=900$ ps [15]. Typical gate fidelities, listed for some rotations in Table \along with the purities, are on the order of $99.5 \%$.

In Fig. 2 we show the corresponding elements of the spin density matrix for rotation $R_{y}(\pi)=$ $R_{z}^{\dagger}(\pi / 2) R_{x}(\pi) R_{z}(\pi / 2)$ acting on the initial spin state $|\bar{x}\rangle$. The $z$ rotations are so fast that on this time scale they appear as vertical steps at $t \simeq 5$ ps and $t \simeq 65 \mathrm{ps}$. The total duration of the gate is also fast, about $60 \mathrm{ps}$. The corresponding spin vector, constructed as $\vec{S}=\operatorname{Tr}\left(\vec{\sigma} \rho_{s}\right)$, is shown in a Bloch sphere in Fig. 3. Here $\vec{\sigma}$ is the spin operator and $\rho_{s}$ is the spin density matrix in the Schrödinger picture.

TABLE I: Fidelity and purity of selected rotations of spin for InAs QD parameters.

\begin{tabular}{|c|c|r|}
\hline$R_{n}(\phi)$ & Fidelity & Purity \\
\hline$R_{x}(\pi / 2)$ & $99.49 \%$ & $98.89 \%$ \\
$R_{z}(\pi / 2)$ & $99.99 \%$ & $99.97 \%$ \\
$R_{y}(\pi)$ & $99.28 \%$ & $98.57 \%$ \\
$R_{y}(\pi / 2)$ & $99.45 \%$ & $98.8 \%$ \\
\hline
\end{tabular}

Our gate scheme is especially attractive for experimental demonstration of optical spin rotations in these systems because it uses only simple, nonadiabatic sech pulses. It does not require phase locking of the lasers in this parameter regime, which is a significant experimental simplification. It requires the ability to perform Rabi oscillations between the spin and the trion, which has been demonstrated recently for InAs QDs [16], and thus it is experimentally accessible with state of the art technology. The only approximation in our method is that the spin is considered to be 'frozen' during the pulse for $z$ rotations, i.e., $\beta_{z} \gg 2\left(\omega_{e}+\omega_{h}\right)$. This requirement can be satisfied for the widely used ultrafast lasers and the current generation of InAs QDs.

For QDs with larger Zeeman splittings the pulse durations discussed above can become too short for practical purposes. Examples of such systems include some CdSe QDs. For such systems our approach can be modified. We utilize the phenomenon of Coherent Population Trapping (CPT), in which a superposition of two levels, each level having nonzero dipole coupling to an excited state, is dark to an (appropriately chosen) coherent combination of laser pulses. This well-known phenomenon in the optics of atoms 17] has recently been demonstrated in a semiconductor [18] for a similar system to the one we study. Here we use frequency selectivity to isolate a three-level system from the four levels of Fig. 1, We do this by choosing narrowband pulses, $\beta \ll 2\left(\omega_{e}-\omega_{h}\right)$, where $\beta$ is the bandwidth. For concreteness we spectrally focus the pulses to pick out the $\Lambda$ system consisting of $\left\{|x\rangle,|\bar{x}\rangle,\left|T_{x}\right\rangle\right\}$. The two transitions are addressed separately by polarization selectivity. The total laser field is

$$
\vec{E}=E_{x} f_{x}(t) e^{i \omega_{x} t} \hat{x}+e^{i \alpha} E_{y} f_{y}(t) e^{i \omega_{y} t} \hat{y}+\text { c.c. }
$$

To create a coherently trapped state the two pulses should have the same detuning (two-photon resonance), i.e., $\omega_{x, T_{x}}-\omega_{x}=\omega_{\bar{x}, T_{x}}-\omega_{y} \equiv \Delta$, and the same temporal envelope $f_{x}(t)=f_{y}(t) \equiv f(t)$. Then the new spin states, $|B\rangle$ and $|D\rangle$ (bright and dark respectively), are related to the basis states $\{|x\rangle,|\bar{x}\rangle\}$ through the unitary transformation

$$
\mathcal{T}=\left[\begin{array}{cc}
\cos \vartheta & -e^{i \alpha} \sin \vartheta \\
e^{-i \alpha} \sin \vartheta & \cos \vartheta
\end{array}\right]
$$

Here $\vartheta$ is defined by

$$
\tan \vartheta=E_{y} / E_{x}
$$

and the matrix element $V_{B, T_{x}}$ between $|B\rangle$ and $\left|T_{x}\right\rangle$ is

$$
V_{B, T_{x}}=\Omega_{o} f(t) e^{i \Delta t},
$$

where $\Omega_{o}=\sqrt{\Omega_{x}^{2}+\Omega_{y}^{2}}[19]$ and $\Omega_{x}\left(\Omega_{y}\right)$ is the Rabi frequency of the transition with polarization $\pi_{x}\left(\pi_{y}\right)$. Now we choose the envelope to be $f(t)=\operatorname{sech}(\beta t)$. We require the population to return to the spin subspace after the passage of the pulse, i.e., we require the total pulse acting on the bright state to be a $2 \pi$ pulse, which gives

$$
\Omega_{o}=\beta
$$

We still have freedom in choosing the bandwidth $\beta$ and the detuning $\Delta$. The bandwidth will be constrained by the requirement of frequency selectivity, $\beta \ll 2\left(\omega_{e}-\omega_{h}\right)$. After the passage of the pulse the bright state has picked up a phase $\phi$ relative to the dark state given by [11]

$$
\phi=2 \arctan \left(\frac{\beta}{\Delta}\right) .
$$

This phase is the angle by which any spin state not parallel to $|B\rangle$ (or, equivalently, $|D\rangle$ ) is rotated. The axis of rotation is that defined by $|B\rangle$. By varying the laser parameters, $\vartheta$ and $\alpha$, we vary the composition of the bright/dark basis, i.e., vary the axis of rotation. Thus we have designed an arbitrary spin rotation

$$
R_{n}(\phi)=e^{-i \phi \hat{n} \cdot \vec{\sigma} / 2},
$$

where $\vec{\sigma}$ is the spin operator. $\phi$ is given by Eq. (8) and the axis of rotation by

$$
\hat{n}=(\cos \vartheta, \sin \vartheta \sin \alpha, \sin \vartheta \cos \alpha) .
$$




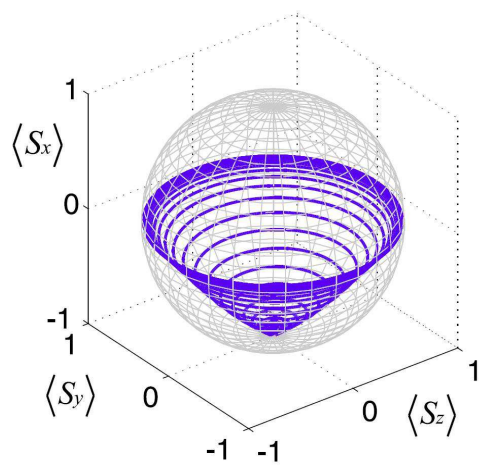

FIG. 4: (Color online) Bloch sphere representation of the spin rotation $R_{z}(\pi / 2)$ acting on the initial spin state $|\bar{x}\rangle$, i.e., $\left\langle S_{x}\right\rangle=-1$.

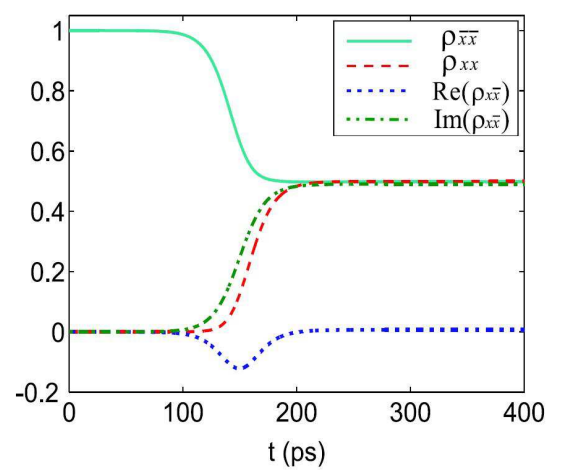

FIG. 5: (Color online) The matrix elements of the density matrix corresponding to $R_{z}(\pi / 2)$, as in Fig. 4. The red (dashed) line is $\rho_{x x}$, the cyan (solid) line is $\rho_{\bar{x} \bar{x}}$, the blue (dotted) line is $\operatorname{Re}\left(\rho_{x \bar{x}}\right)$, the green (dashed-dotted) line is $\operatorname{Im}\left(\rho_{x \bar{x}}\right) . \quad \rho$ is the density matrix in the interaction picture. The duration of this gate is about 100 ps.

This axis is the same as that of the Kis and Renzoni scheme [9] where CPT also is used. However, in their approach an auxiliary lower level is required for the population to be stored during the gate. This extra requirement limits the applicability of their scheme to physical systems where such an extra level is available. In our case, no auxiliary levels are needed, and the same pulse creates the trapped state and induces the geometric phase.

The main sources of dissipation in the CPT-based scheme are non-resonant transitions to the higher trion state $\left|T_{\bar{x}}\right\rangle$ and spontaneous emission of the trion. To determine their effects on the gates we have made numerical simulations of the fidelity. We choose parameters appropriate for CdSe nanocrystals as an example of a physical system with relatively large Zeeman splittings: $\omega_{e}=0.4 \mathrm{meV}, \omega_{h}=0.05 \mathrm{meV}$ (corresponding to $\mathrm{B} \sim 8$ $\mathrm{T}), \beta \sim 0.025 \mathrm{meV}$, and trion lifetime $\tau_{t}=580 \mathrm{ps}[20]$. We find that for gate durations of about 100 ps typical fidelities range between $98 \%$ and $99 \%$, and they are shown in Table II. A Bloch sphere representation of the spin rotation $R_{z}(\pi / 2)$ is shown in Fig. 4, and the corresponding spin density matrix elements are plotted as functions of time in Fig. 5. This figure shows the total duration of the gate and also shows that the population $\left(\rho_{x x}+\rho_{\bar{x} \bar{x}}\right)$ remains mostly in the spin subspace during the greatest part of the gate.

The above CPT scheme is an exact analytical solution for a true three-level $\Lambda$ system. We note that as the Zeeman splitting of either the electron or the trion gets larger the system more closely approaches a $\Lambda$ system. In that case fast pulses can be used to beat the excited state decay time, and the fidelities then approach $100 \%$. This rotation scheme may also be used to implement gates in QIP with other quantum systems such as ions or atoms where the excited states are typically longer lived than those of QDs.

TABLE II: Fidelity and purity of selected rotations for CdSe QD parameters.

\begin{tabular}{|c|c|r|}
\hline$R_{n}(\phi)$ & Fidelity & Purity \\
\hline$R_{z}(\pi / 2)$ & $98.84 \%$ & $97.8 \%$ \\
$R_{z}(\pi)$ & $97.56 \%$ & $95.34 \%$ \\
$R_{x}(\pi / 2)$ & $98.42 \%$ & $97 \%$ \\
$R_{x z}(\pi / 2)$ & $99.2 \%$ & $98.78 \%$ \\
\hline
\end{tabular}

In conclusion, we have developed a method of realizing the key step of optical spin rotations in realistic QDs based on the geometric phases induced by hyperbolic secant pulses of area $2 \pi$. They are used with either dark or coherently trapped states, depending on the parameters of the QDs. This scheme should be accessible with current experimental capabilities, and it yields fast (1ps-100 ps) rotations because it does not employ the adiabatic approximation. The fidelities of these rotations for typical QD systems are high, up to $99.99 \%$. Our method provides an exact solution to rotations in a threelevel $\Lambda$-type system and may prove useful to other areas of physics that involve interaction of coherent radiation with quantum systems [22].

This work is supported by the US Office of Naval Research. One of us (S.E.E.) is an NRC/NRL Research Associate.

[1] A. Imamoğlu, D. D. Awschalom, G. Burkard, D. P. DiVincenzo, D. Loss, M. Sherwin, and A. Small, Phys. Rev. Lett. 83, 4204 (1999).

[2] D. P. DiVincenzo, Fortschr. Phys. 48, 771 (2000).

[3] M. V. G. Dutt, J. Cheng, B. Li, X. Xu, X. Li, P. R. Berman, D. G. Steel, A. S. Bracker, D. Gammon, S. E. Economou, et al., Phys. Rev. Lett. 94, 227403 (2005).

[4] S. Cortez, O. Krebs, S. Laurent, M. Senes, X. Marie, P. Voisin, R. Ferreira, G. Bastard, J.-M. Gèrard, and T. Amand, Phys. Rev. Lett. 89, 207401 (2002). 
[5] M. Atatüre, J. Dreiser, A. Badolato, and A. Imamoğlu, Nature 3, 101 (2007).

[6] A. Greilich, D. R. Yakovlev, A. Shabaev, A. L. Efros, I. A. Yugova, R. Oulton, V. Stavarache, D. Reuter, A. Wieck, and M. Bayer, Science 313, 341 (2006).

[7] P. Chen, C. Piermarocchi, L. J. Sham, D. Gammon, and D. G. Steel, Phys. Rev. B 69, 075320 (2003).

[8] C. Emary and L. J. Sham, arXiv:cond-mat/0608518 (2006).

[9] Z. Kis and F. Renzoni, Phys. Rev. A 65, 032318 (2002).

[10] F. Troiani, E. Molinari, and U. Hohenester, Phys. Rev. Lett. 90,206802 (2003).

[11] S. E. Economou, L. J. Sham, Y. Wu, and D. G. Steel, Phys. Rev. B 74, 205415 (2006).

[12] N. Rosen and C. Zener, Phys. Rev. 40, 502 (1932).

[13] S. McCall and E. Hahn, Phys. Rev. 183 (1969).

[14] M. D. Bowdrey, D. K. Oi, A. J. Short, K. Banaszek, and J. A. Jones, Phys. Lett. A 294, 258 (2002).

[15] M. E. Ware, E. A. Stinaff, D. Gammon, M. F. Doty, A. S. Bracker, D. Gershoni, V. L. Korenev, S. C. Bădescu, Y. Lyanda-Geller, and T. L. Reinecke, Phys. Rev. Lett. 95, 177403 (2005).

[16] A. Greilich, R. Oulton, E. A. Zhukov, I. A. Yugova,
D. R. Yakovlev, M. Bayer, A. Shabaev, A. L. Efros, I. A. Merkulov, V. Stavarache, et al., Phys. Rev. Lett. 96, 227401 (2006).

[17] G. Alzetta, A. Gozzini, L. Moi, and G. Orriols, Nuovo Cimento B 36, 5 (1976).

[18] K.-M. C. Fu, C. Santori, C. Stanley, M. Holland, and Y. Yamamoto, Phys. Rev. Lett. 95, 187405 (2005).

[19] J. H. Eberly and V. V. Kozlov, Phys. Rev. Lett. 88, 243604 (2002).

[20] J. Seufert, G. Bacher, M. Scheibner, A. Forchel, S. Lee, M. Dobrowolska, and J. K. Furdyna, Phys. Rev. Lett. 88, 027402 (2002).

[21] G. Panzarini, U. Hohenester, and E. Molinari, Phys. Rev. B 65, 165322 (2002).

[22] An additional advantage of our scheme is that the use of $2 \pi$ sech pulses may allow for potential manipulation by the same pulse of a logical qubit comprised by several physical qubits located along the optical path. Then, as in self-induced transparency, the different qubits would interact with a single reshaped pulse. We note that numerical simulations 21] imply that self-induced transparency is possible in an array of QDs. 